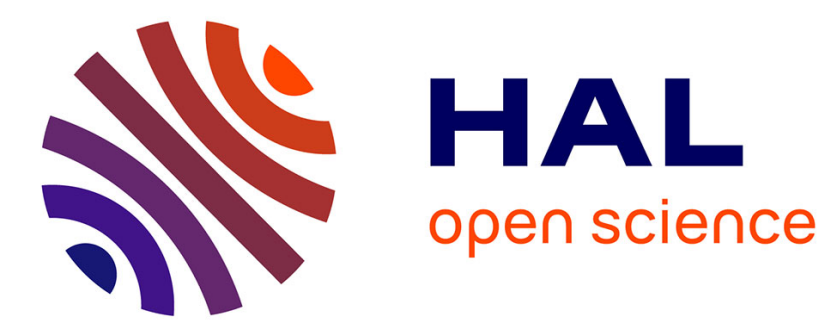

\title{
Absolute and convective instabilities of a swirling jet/wake shear layer
}

Thomas Loiseleux, Ivan Delbende, Patrick Huerre

\section{To cite this version:}

Thomas Loiseleux, Ivan Delbende, Patrick Huerre. Absolute and convective instabilities of a swirling jet/wake shear layer. Physics of Fluids, 2000, 12 (2), pp.375-380. 10.1063/1.870315 . hal-01025355

\section{HAL Id: hal-01025355 \\ https://hal-polytechnique.archives-ouvertes.fr/hal-01025355}

Submitted on 11 Sep 2014

HAL is a multi-disciplinary open access archive for the deposit and dissemination of scientific research documents, whether they are published or not. The documents may come from teaching and research institutions in France or abroad, or from public or private research centers.
L'archive ouverte pluridisciplinaire HAL, est destinée au dépôt et à la diffusion de documents scientifiques de niveau recherche, publiés ou non, émanant des établissements d'enseignement et de recherche français ou étrangers, des laboratoires publics ou privés. 


\section{AIP | Physics of Fluids}

\section{Absolute and convective instabilities of a swirling jet/wake shear layer}

Thomas Loiseleux, Ivan Delbende, and Patrick Huerre

Citation: Physics of Fluids (1994-present) 12, 375 (2000); doi: 10.1063/1.870315

View online: http://dx.doi.org/10.1063/1.870315

View Table of Contents: http://scitation.aip.org/content/aip/journal/pof2/12/2?ver=pdfcov

Published by the AIP Publishing

\section{Articles you may be interested in}

Experimental and numerical investigation of a variable density swirling-jet stability

Phys. Fluids 25, 084104 (2013); 10.1063/1.4817070

Inviscid instability of the Batchelor vortex: Absolute-convective transition and spatial branches

Phys. Fluids 11, 1805 (1999); 10.1063/1.870045

Nonlinear convective/absolute instabilities in parallel two-dimensional wakes

Phys. Fluids 10, 2724 (1998); 10.1063/1.869796

The effect of swirl on jets and wakes: Linear instability of the Rankine vortex with axial flow

Phys. Fluids 10, 1120 (1998); 10.1063/1.869637

Absolute/convective transition of wake-dominated supersonic shear layers

Phys. Fluids 9, 679 (1997); 10.1063/1.869225

\section{A|P| $\left.\right|_{\text {Applied Physics }} ^{\text {Journal of }}$}

Journal of Applied Physics is pleased to announce André Anders as its new Editor-in-Chief 


\title{
Absolute and convective instabilities of a swirling jet/wake shear layer
}

\author{
Thomas Loiseleux, ${ }^{\text {a) }}$ Ivan Delbende, ${ }^{\text {b) }}$ and Patrick Huerre \\ Laboratoire d'Hydrodynamique, CNRS-Ecole polytechnique, F-91128 Palaiseau, France
}

(Received 30 October 1998; accepted 21 October 1999)

\begin{abstract}
The absolute (AI)/convective (CI) nature of the instability is determined in the family of swirling jet/wake shear layers considered by Martin and Meiburg [Phys. Fluids 6, 424 (1994)] and Lim and Redekopp [Eur. J. Mech. B/Fluids 17, 165 (1998)]. This idealized model includes as essential ingredients both the centrifugal instability associated with the swirl difference and the KelvinHelmholtz instability associated with the swirl and axial velocity differences between the core and the outer flow. Centrifugally stabilizing or destabilizing swirl differences are found to promote AI, but a centrifugally destabilizing configuration is more effective in triggering such a transition. For sufficiently large swirl differences, both co-flowing jets and wakes may become AI. In the case of jets, a centrifugally destabilizing swirl difference first brings about AI via the axisymmetric mode $m=0$ in a large range of mean swirl values. By contrast, a centrifugally stabilizing swirl difference triggers AI via the helical mode $m=-\infty$. In the case of wakes, a centrifugally destabilizing swirl difference leads to AI via the bending mode $m=1$ whereas a centrifugally stabilizing swirl difference triggers AI via various negative helical modes $m=-1,-2$, etc. (C) 2000 American Institute of Physics. [S1070-6631(00)01102-8]
\end{abstract}

\section{INTRODUCTION}

The pervasiveness of vortical flows in nature makes their description and control an important issue in fluid mechanics research. Understanding such phenomena as water-spouts and tornadoes in meteorology, leading-edge and wing-tip vortices in aerodynamics has motivated numerous studies dealing with the structure, stability, and breakdown of swirling flows. In swirling jets and wakes, dynamical modifications may be ascribed to a change in the nature of the instability from convective to absolute, i.e., to the emergence of an instability wave with a zero-group velocity. ${ }^{1,2}$ More precisely, such a transition may explain some of the features associated with the onset of vortex breakdown. In this spirit, previous studies have addressed the absolute/convective instability problem for two specific swirling flow models: The Batchelor vortex $^{3-5}$ and the Rankine vortex with axial flow. ${ }^{6}$ In both instances, the circulation of the base flow monotonically increases with radial distance so that, according to the Rayleigh criterion, ${ }^{7,8}$ centrifugal instabilities are quenched. This is not necessarily the case in many practical or experimental situations, i.e., wing-tip vortices ${ }^{9}$ or swirling jet experiments, ${ }^{10}$ where the total circulation remains zero at large radial distances. In such cases, the circulation decreases with radial distance over part of the flow, a feature which favors the onset of centrifugal instabilities. In the present article, the interaction and competition between axial shear and centrifugal instabilities are qualitatively assessed on the basis of a simple analytical model. We thus determine the absolute/convective instability characteristics of a family of

\footnotetext{
${ }^{a)}$ Present address: ENSTA, 32 Boulevard Victor, 75739 Paris Cedex 15, France. Electronic mail: thomas.loiseleux@ensta.fr

b) Present address: LIMSI, Université de Paris-Sud, 91403 Orsay Cedex, France. Electronic mail: delbende@limsi.fr
}

swirling jet/wake shear layers consisting of a straight vortex filament of circulation $\Gamma_{c}$ surrounded by a cylindrical vortex sheet of strength $\Gamma_{c}-\Gamma_{\infty}$, where $\Gamma_{\infty}$ is the external circulation. A plug flow axial jet/wake velocity profile (Fig. 1) is added to this purely swirling cylindrical vortex sheet. According to Hall ${ }^{11}$ the azimuthal velocity radial structure of a leading-edge vortex can be approximated as a thin viscous sub-core embedded within an otherwise inviscid irrotational outer flow. The base flow in Fig. 1 should be viewed as a simple canonical model of such physical situations.

Martin and Meiburg ${ }^{12}$ have determined the linear temporal instability properties of this family of profiles: For centrifugally stabilizing flows $\left(\Gamma_{c}-\Gamma_{\infty}<0\right)$, the instability is of Kelvin-Helmholtz-type, feeding on both azimuthal and axial vorticity, and counter-rotating helical waves $(m<0)$ are the most unstable. When the flow is centrifugally destabilizing $\left(\Gamma_{c}-\Gamma_{\infty}>0\right)$, the most unstable modes consist of corotating helical waves $(m>0)$. These properties are in qualitative agreement with physical intuition: Kelvin-Helmholtz instability generates cross-stream vortices with a wave vector $(k, m)$ perpendicular to the local shear, which therefore, take the shape of counter-rotating spirals $(m<0)$, whereas centrifugal instability generates locally streamwise vortices spiralling with the flow $(m>0)$. It should be made clear that this model does not take into consideration the finite thickness of the cylindrical shear layer: Arbitrarily large streamwise and azimuthal wave numbers remain unstable. Under such conditions, the temporal instability framework does not single out a most amplified mode.

Kang and $\operatorname{Lin}^{13}$ and Lian and $\operatorname{Lin}^{14}$ have investigated the spatial instability characteristics of a swirling or nonswirling liquid jet emanating in a nonswirling or swirling gas in order to gain a better understanding of the break-up process. Their velocity fields correspond to the limiting cases $\Gamma_{\infty}=0$ in Ref. 


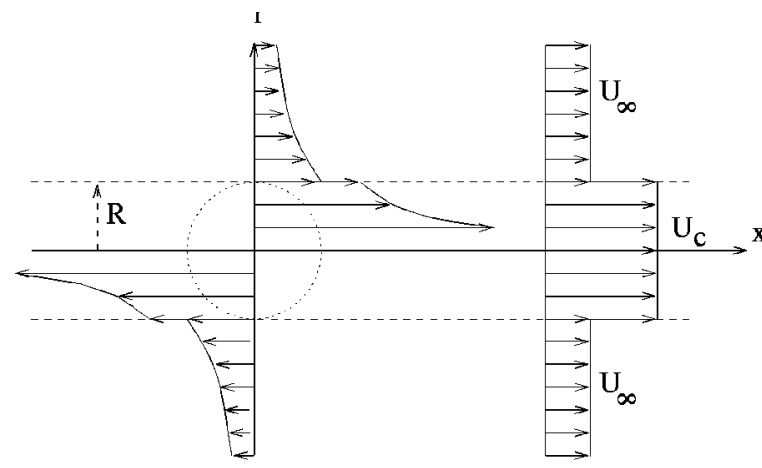

FIG. 1. Sketch of basic flow azimuthal and axial velocity profiles.

13 and $\Gamma_{c}=0$ in Ref. 14 of the present flow model. A destabilizing (resp. stabilizing) effect of swirl was identified for the centrifugally destabilizing (resp. stabilizing) configuration $\Gamma_{\infty}=0$ (resp. $\left.\Gamma_{c}=0\right)$. An increasing gas-to-liquid ratio was determined to be destabilizing in the case of a swirling jet. ${ }^{13}$ It was found to be stabilizing or destabilizing in the case of a liquid jet in a swirling gas, ${ }^{14}$ depending on the respective magnitudes of the density ratio and Weber number. In the present study we concentrate our attention on the instability properties of a homogeneous fluid without surface tension and density variations.

The absolute/convective instability characteristics of several families of swirling jets and wakes have recently been examined by analytical and numerical means. The centrifugally stabilizing continuous basic flow commonly referred to as the Batchelor vortex is known to provide an approximation of the velocity field within trailing line vortices. Olendraru et al., ${ }^{3,5}$ have applied the zero-group velocity criterion of Briggs and Bers ${ }^{1,2}$ to the numerically determined dispersion relation of the Batchelor vortex. These issues have also been examined for the same basic flow by direct numerical simulations of the linear impulse response (Delbende, Chomaz, and Huerre ${ }^{4}$ ). According to these studies, swirl greatly enhances the range of absolute instability to the extent that, for intermediate swirl values, co-flowing jets and wakes become absolutely unstable. The transitional modes are all negative but take distinct values for jets and wakes. The piecewise continuous Rankine vortex with superimposed axial plug flow constitutes an idealized representation of the velocity field prevailing close to the nozzle exit in swirling jets in the absence of a centrifugally destabilizing region. As in the previous case, Loiseleux Chomaz and Huerre $^{6}$ have shown that the application of swirl promotes the AI of negative helical modes of increasingly higher order. The absence of a finite shear layer thickness then prevents the quenching of large streamwise and azimuthal wave numbers.

The absolute/convective instability characteristics of two idealized models of centrifugally destabilizing swirling flows have recently been analyzed by Lim and Redekopp. ${ }^{15}$ In the first model, a modified Rankine vortex with superimposed axial flow is allowed to exhibit a centrifugally destabilizing azimuthal velocity discontinuity. The increasing magnitude of the discontinuity is then shown to very significantly en- hance the absolute growth rate of the axisymmetric mode. Decreasing the core density, with respect to the external density, also contributes to increase the absolute growth rate. The second model is the same as in the present study: In the uniform density case, jets are shown to undergo absolute instability via the $m=0$ mode as the ratio of the external to core swirl is decreased, thereby enhancing the centrifugal instability. Furthermore, decreasing the core density, with respect to the external density, tends to promote AI. When this second model is analyzed in the wake configuration, it is concluded that the negative helical modes are more absolutely unstable than their positive counterparts. It is argued that centrifugally destabilizing swirl promotes AI whereas centrifugally stabilizing swirl inhibits it.

The goal of the present study, which solely focuses on the uniform density case, is to systematically determine the absolute/convective instability boundary of the basic flow displayed in Fig. 1 by locating the absolute/convective transition curves of all positive and negative helical modes. A complete state diagram is obtained for co-flowing or counterflowing jets and wakes and for centrifugally destabilizing or stabilizing swirl differences across the cylindrical vortex sheet. As summarized in the conclusion, the results very significantly extend those obtained by Lim and Redekopp ${ }^{15}$ for their second model. The analysis of higher order positive or negative helical modes is crucial to the determination of the absolute/convective instability boundary of the flow and the modes examined by Lim and Redekopp ${ }^{15}$ are found not to be the critical ones in several basic flow configurations.

\section{PROBLEM FORMULATION AND LINEAR DISPERSION RELATION}

In the entire study, the flow is assumed to be inviscid and incompressible. As sketched in Fig. 1, the basic flow under consideration consists of a straight vortex filament aligned along the $x$ axis surrounded by a co-axial cylindrical vortical sheet of radius $R$, upon which is superimposed a plug flow axial velocity profile. Let $U$ and $W$ denote the axial and azimuthal velocity components in the usual $x-r-\theta$ cylindrical coordinate system. The basic velocity field is then given by

$$
\begin{aligned}
& U(r)=U_{c}, \quad W(r)=\frac{\Gamma_{c}}{2 \pi r}, \quad \text { if } r<R, \\
& U(r)=U_{\infty}, \quad W(r)=\frac{\Gamma_{\infty}}{2 \pi r}, \quad \text { if } r>R,
\end{aligned}
$$

where $U_{c}, \Gamma_{c}$ are the core axial velocity and circulation, and $U_{\infty}, \Gamma_{\infty}$ the external axial velocity and circulation. The basic flow effectively depends on three nondimensional control parameters, for instance, the external flow $a$ and the normalized core and external circulations $\gamma_{c}$ and $\gamma_{\infty}$ defined as

$$
a \equiv \frac{U_{\infty}}{\Delta U}, \quad \gamma_{c} \equiv \frac{\Gamma_{c}}{R \Delta U}, \quad \gamma_{\infty} \equiv \frac{\Gamma_{\infty}}{R \Delta U},
$$

where $\Delta U=U_{c}-U_{\infty}$ represents the axial shear. This set of parameters based on the reference scales $\Delta U$ and $R$ is well suited to discuss the effects of rotation on the instability 


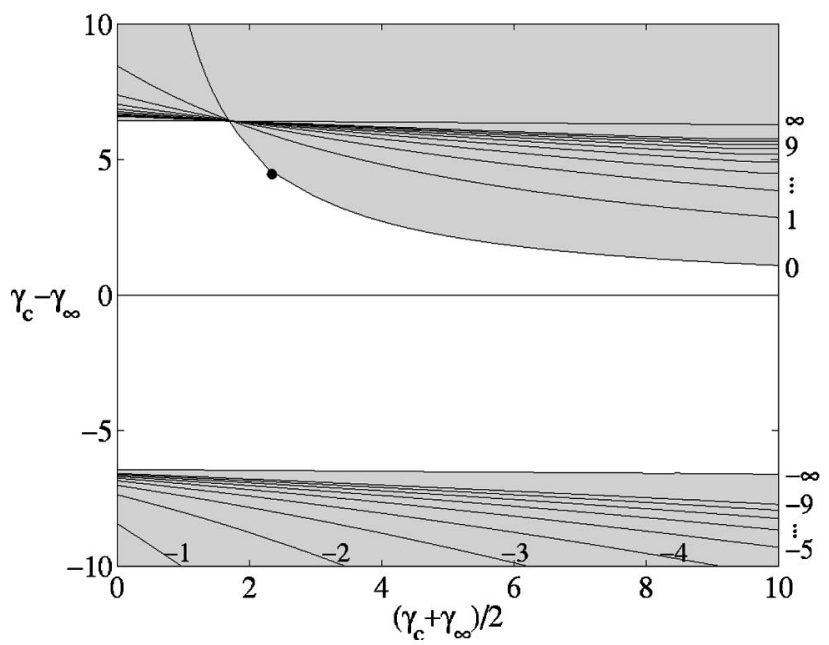

(a)

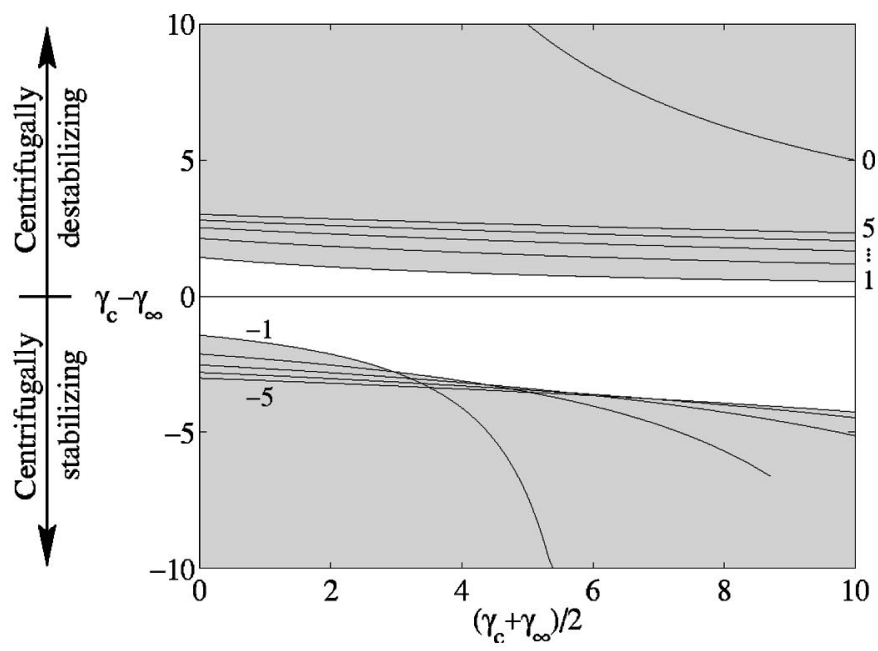

(b)

FIG. 2. AI/CI domains in the $\left(\gamma_{c}+\gamma_{\infty}\right) / 2-\left(\gamma_{c}-\gamma_{\infty}\right)$ plane for (a) a zero-external axial flow jet $(a=0)$ and (b) a zero-centerline velocity wake $(a=-1)$. The shaded area corresponds to the absolutely unstable region, and the clear area to the convectively unstable region. The line $\gamma_{c}-\gamma_{\infty}=0$, separates the centrifugally stabilizing region $\left(\gamma_{c}-\gamma_{\infty}<0\right)$ and the centrifugally destabilizing region $\left(\gamma_{c}-\gamma_{\infty}>0\right)$. The solid dot in (a) corresponds to the AI/CI transition point $\gamma_{c}=4.65, \gamma_{\infty}=0$ calculated in Ref. 15 .

characteristics of plug flow axial jets and wakes. A contrario, if one is interested in the effect of axial shear and advection on the instability characteristics of cylindrical azimuthal vortex sheets, it is more appropriate to use as reference scales $\Delta \Gamma=\Gamma_{c}-\Gamma_{\infty}$ and $R$. An alternate set of nondimensional control parameters may then be defined as:

$$
\delta \gamma \equiv \frac{\Delta \Gamma}{\Gamma_{\infty}}, \quad \delta u \equiv \frac{R \Delta U}{\Delta \Gamma}, \quad \bar{u} \equiv \frac{R\left(U_{c}+U_{\infty}\right)}{2 \Delta \Gamma} .
$$

In most of the study, results are presented in terms of the set of parameters (2). The set (3) is briefly used at the end of the paper to illustrate the influence of axial shear $\delta u$ and advection $\bar{u}$.

As discussed for instance in Loiseleux, Chomaz and Huerre (see Fig. 2 of Ref. 8), distinct axial velocity configurations are obtained for specific ranges of $a$. The external and core streams are co-flowing when $a<-1$ or $a>0$ and counter-flowing when $-1<a<0$. Jets correspond to $a>-0.5$ and wakes to $a<-0.5$. The particular values $a=0$ and $a=-1$ are associated with the zero external flow jet and zero centerline velocity wake respectively.

Following Martin and Meiburg, ${ }^{1}$ the nondimensional linear dispersion relation is readily found to be

$$
\begin{aligned}
\omega= & \Omega\left(k ; a, \gamma_{c}, \gamma_{\infty}, m\right) \\
= & a k+\frac{1}{\alpha-\beta}\left(\frac{m}{2 \pi}\left(\alpha \gamma_{c}-\beta \gamma_{\infty}\right)+k \alpha\right) \\
& \pm i\left[\frac { 1 } { 4 ( \alpha - \beta ) } \left(\frac{4 m k \alpha \gamma_{c}}{\pi}+\frac{m^{2}}{\pi^{2}}\left(\alpha \gamma_{c}^{2}-\beta \gamma_{\infty}^{2}\right)+4 k^{2} \alpha\right.\right. \\
& \left.\left.-\frac{\gamma_{\infty}^{2}-\gamma_{c}^{2}}{\pi^{2}}\right)-\frac{1}{(\alpha-\beta)^{2}}\left(\frac{m}{2 \pi}\left(\alpha \gamma_{c}-\beta \gamma_{\infty}\right)+k \alpha\right)^{2}\right]^{1 / 2},
\end{aligned}
$$

where

$$
\alpha(k) \equiv \frac{I_{m}(k)}{k I_{m}^{\prime}(k)} \quad \text { and } \beta(k) \equiv \frac{K_{m}(k)}{k K_{m}^{\prime}(k)}
$$

\section{ABSOLUTE/CONVECTIVE INSTABILITY CHARACTERISTICS}

As extensively discussed in the literature (see for instance Bers ${ }^{2}$ and Huerre and Monkewitz ${ }^{16}$ ), the absolute/ convective nature of the instability is determined by the sign of the absolute growth rate $\omega_{0, i}$, associated with the saddle points of the dispersion relation satisfying

$$
\left\{\begin{array}{l}
\frac{d \Omega}{d k}\left(k_{0} ; a, \gamma_{c}, \gamma_{\infty}, m\right)=0 \\
\omega_{0}=\Omega\left(k_{0} ; a, \gamma_{c}, \gamma_{\infty}, m\right) .
\end{array}\right.
$$

In addition the saddle points should emerge from the pinching of two spatial branches issuing from the upper and lower parts of the complex $k$-plane. If $\omega_{0, i}>0\left(\omega_{0, i}<0\right)$ the flow is absolutely unstable (resp. convectively unstable). Details of the numerical procedure used to determine $\omega_{0}$ may be found in Loiseleux et al. ${ }^{6}$

The diagrams in Fig. 2 document the effect of the swirl difference $\gamma_{c}-\gamma_{\infty}$ and of the mean swirl $\left(\gamma_{c}+\gamma_{\infty}\right) / 2$ on the absolute/convective nature of the instability in zero-external axial flow jets $[a=0$, Fig. 2(a) $]$ and zero-centerline velocity wakes $[a=-1$, Fig. 2(b)].

Consider first the case $a=0$ [Fig. 2(a)]. In the absence of rotation $\left(\gamma_{c}=\gamma_{\infty}=0\right)$, zero-external axial flow jets are convectively unstable. In order to discuss the effect of swirl, it is convenient to distinguish in the $\left(\gamma_{c}+\gamma_{\infty}\right) / 2-\left(\gamma_{c}-\gamma_{\infty}\right)$ plane the centrifugally destabilizing and stabilizing domains $\gamma_{c}-\gamma_{\infty}>0$ and $\gamma_{c}-\gamma_{\infty}<0$, respectively. The application of a sufficiently large centrifugally destabilizing swirl difference $\gamma_{c}-\gamma_{\infty}>0$ is seen to induce transition to AI via the axisymmetric mode $m=0$ whenever the mean swirl $\left(\gamma_{c}\right.$ 


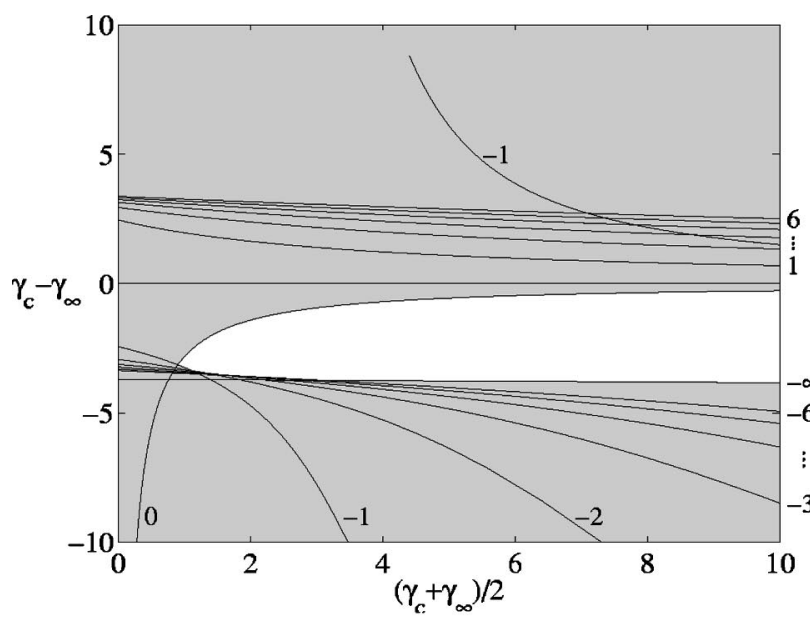

(a)

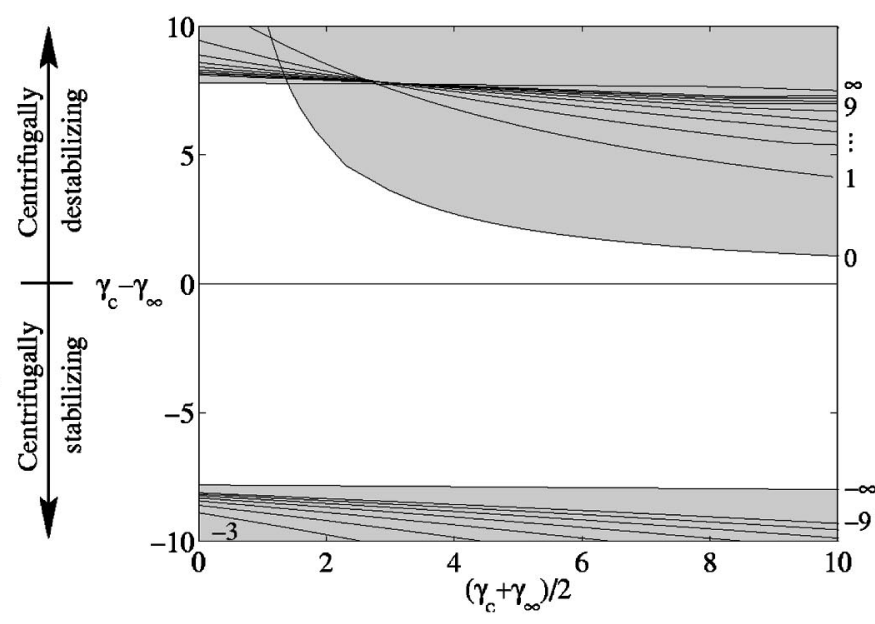

(b)

FIG. 3. AI/CI domains in the $\left(\gamma_{c}+\gamma_{\infty}\right) / 2-\left(\gamma_{c}-\gamma_{\infty}\right)$ plane for (a) an external counterflow jet at $a=-0.2$ and (b) an external co-flow jet at $a=0.1$. The shaded area corresponds to the absolutely unstable region, and the clear area to the convectively unstable region. The line $\gamma_{c}-\gamma_{\infty}=0$ separates the centrifugally stabilizing region $\left(\gamma_{c}-\gamma_{\infty}<0\right)$ and the centrifugally destabilizing region $\left(\gamma_{c}-\gamma_{\infty}>0\right)$.

$\left.+\gamma_{\infty}\right) / 2$ exceeds 1.7. When $\left(\gamma_{c}+\gamma_{\infty}\right) / 2$ is lower than 1.7 , transition to AI occurs via $m=+\infty$. By contrast, a centrifugally stabilizing swirl difference $\gamma_{c}-\gamma_{\infty}<0$ always provokes a transition to AI via the pathological helical mode $m=-\infty$. This unusual feature has been verified numerically: For a fixed $\left(\gamma_{c}+\gamma_{\infty}\right) / 2$, the AI/CI transition value of $\gamma_{c}-\gamma_{\infty}$ pertaining to a given negative helical mode $m$ increases continuously towards an asymptotic maximum as $m$ tends to $-\infty$. The locus of such maxima as $\left(\gamma_{c}+\gamma_{\infty}\right) / 2$ is varied constitutes the curve labeled $m=-\infty$ in Fig. 2(a). The numerical value of $m=-\infty$ is taken to be $m=-40$. The same methodology is used to obtain the AI/CI transition curve labeled $m=+\infty$ on the centrifugally destabilizing side $\gamma_{c}-\gamma_{\infty}>0$. According to Fig. 2(a), a centrifugally destabilizing swirl difference is more efficient in triggering a transition to AI than a centrifugally stabilizing swirl difference. More importantly, a centrifugally destabilizing swirl difference clearly promotes axisymmetric or positive helical modes, whereas a centrifugally stabilizing swirl difference promotes negative helical modes of gradually decreasing $|m|$. The AI/CI transition point $\left(\gamma_{c}=4.65 ; \gamma_{\infty}=0\right)$ for the $m=0$ mode in Fig. 2(a) is in satisfactory agreement with the result $\gamma_{c}=2 \pi \times 0.74=4.62$ obtained by Lim and Redekopp ${ }^{2}$ at $\gamma_{\infty}=0$.

Consider next the case $a=-1$ [Fig. 2(b)]. In the absence of rotation $\left(\gamma_{c}=\gamma_{\infty}=0\right)$, the zero-centerline velocity wake is CI. In this instance, a moderately large centrifugally destabilizing swirl difference induces a transition to AI via the positive bending mode $m=1$. A centrifugally stabilizing swirl difference clearly promotes negative helical modes but the critical transitional mode is sensitive to the value of $\left(\gamma_{c}+\gamma_{\infty}\right) / 2$. As in the previous case, a centrifugally destabilizing swirl difference is more effective in promoting AI. At first sight, the present results for $\gamma_{c}-\gamma_{\infty}>0$ do not corroborate those of Lim and Redekopp ${ }^{15}$ for $\gamma_{c}>0$ and $\gamma_{\infty}=0$ : Negative helical modes are found by these authors to be critical whereas the present study clearly singles out positive helical modes. Such a discrepancy is more apparent than real: It is due to the fact that the swirl parameter $S_{w}$ introduced in Ref. 15 is related to the parameters of the present study by $S_{w}=\gamma_{c} / 2 \pi a$. When $a=-1, \gamma_{c}>0$ corresponds to $S_{w}<0$ and consequently $m$ is changed into $-m$. Furthermore, the curves $m=-1$ and $m=-2$ in Fig. 17 of Ref. 15 should probably be switched. If such substitutions are made, there is both quantitative and qualitative agreement between both studies.

It is worth pointing out the peculiar features which arise along the line $\gamma_{c}-\gamma_{\infty}=0$ separating the centrifugally destabilizing and stabilizing domains. Such a configuration corresponds to a single vortex filament embedded in an axial plug flow. The dispersion relation (4) then reduces to

$$
\omega=a k+\frac{m \gamma}{2 \pi}+k \frac{\alpha \pm \sqrt{\alpha \beta}}{\alpha-\beta},
$$

where $\gamma_{c}=\gamma_{\infty}=\gamma$. As a result, the absolute wave number $k_{0}$ and the absolute growth rate $\omega_{0, i}$ do not depend on $\gamma$. The $\mathrm{AI} / \mathrm{CI}$ transition values of $a$ are also $\gamma$-independent and equal to those prevailing at $\gamma=0$, namely $a_{c}=-0.15, m=0$ on the jet side and $a_{c}=-0.91,|m|=1$ on the wake side. ${ }^{6}$ At these specific values of the external axial flow parameter $a$, AI/CI transition takes place simultaneously along the line $\gamma_{c}-\gamma_{\infty}=0$. In other words, a single vortex filament of strength $\gamma$ does not alter the AI/CI characteristics of plug flow velocity jets or wakes.

A typical external counter-flow jet case $(-0.5<a<0)$ is illustrated in Fig. 3(a). When the counterflow $|a|$ is gradually increased, the CI-domain shrinks to a narrow band on the centrifugally stabilizing side $\gamma_{c}-\gamma_{\infty}<0$, and the flow becomes AI over most of the parameter plane. A typical external co-flow jet case $(a>0)$ is illustrated in Fig. 3(b). When the co-flow $a$ is gradually increased, the CI-domain expands and occupies most of the control parameter plane. The same qualitative tendencies hold for counter-flow and co-flow wakes. 


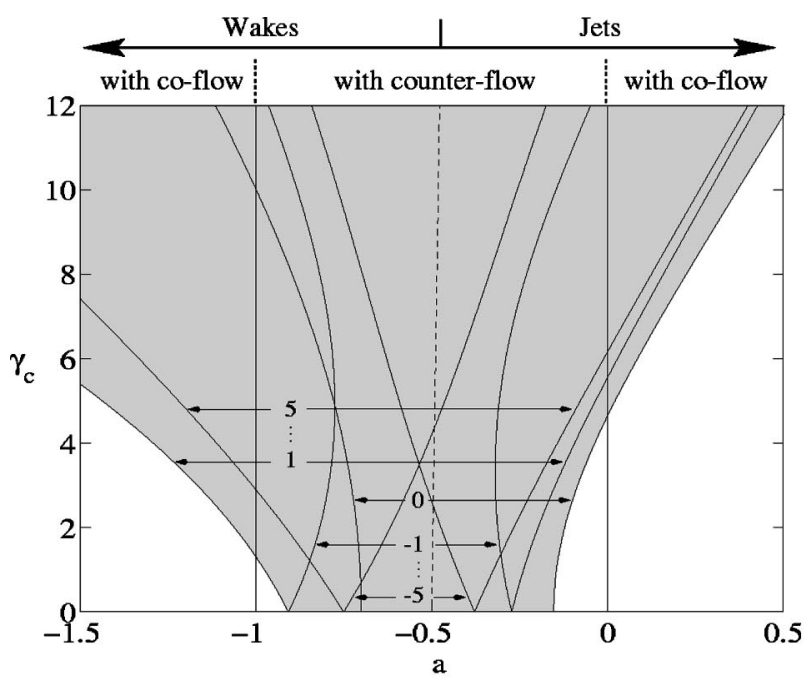

(a)

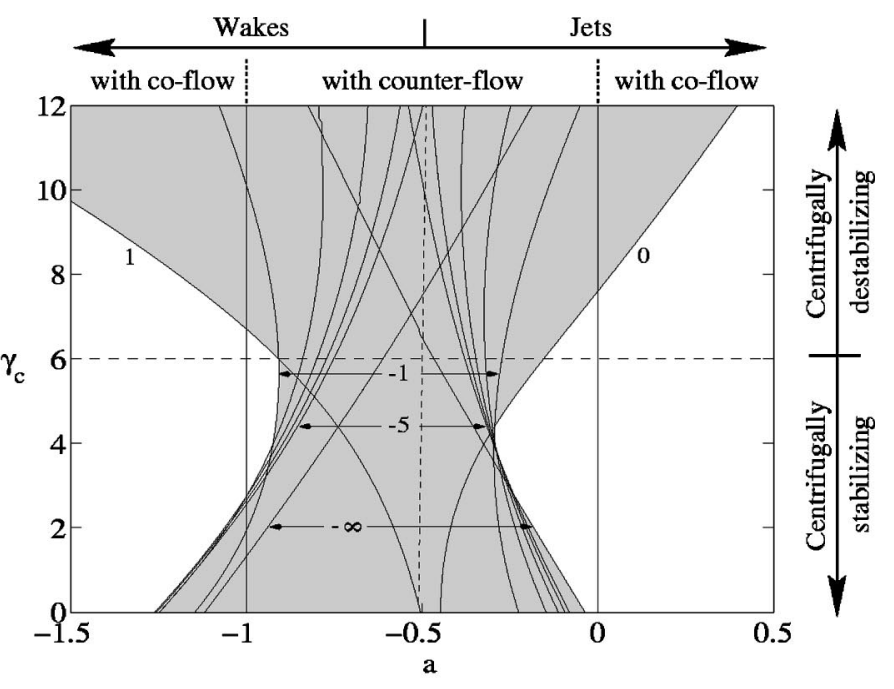

(b)

FIG. 4. AI/CI domains in the $a-\gamma_{c}$ plane for (a) a zero-external swirl $\left(\gamma_{\infty}=0\right)$ and (b) a finite external swirl $\left(\gamma_{\infty}=6\right)$. The shaded area corresponds to the absolutely unstable region, and the clear area to the convectively unstable region. The dotted line $\gamma_{c}=\gamma_{\infty}$ separates the centrifugally stabilizing and destabilizing regions. For clarity, in (b), only the helical modes participating in the delineation of the AI/CI regions have been represented.

To compare the present results with those pertaining to the Rankine vortex with axial flow (Ref. 6), it is enlightening to display the AI/CI domains at a fixed setting of $\gamma_{\infty}$ as shown in Fig. 4. Such diagrams are the equivalent of Fig. 20 in Ref. 6. In the absence of external swirl [ $\gamma_{\infty}=0$, Fig. 4(a) $]$, the effect of a finite $\gamma_{c}$ is always centrifugally destabilizing. The $a$-interval of $\mathrm{AI}$ is seen to increase continuously with the core swirl $\gamma_{c}$. The transitional modes $m=0$ and $m=1$ are clearly in evidence on the jet and wake sides, respectively. For a finite external swirl $\left[\gamma_{\infty}=6\right.$, Fig. 4(b)], the effect of a finite $\gamma_{c}$ is centrifugally stabilizing below $\gamma_{c}=\gamma_{\infty}$ and centrifugally destabilizing above $\gamma_{c}=\gamma_{\infty}$. However, in both cases, the $a$-interval of AI expands with increasing swirl difference $\left|\gamma_{c}-\gamma_{\infty}\right|$. Furthermore, in the centrifugally destabilizing domain $\gamma_{c}>\gamma_{\infty}$, the AI range is bounded by the $m=0$ and $m=1$ modes on the jet and wake sides, respectively. In the centrifugally stabilizing domain $\gamma_{c}<\gamma_{\infty}$, various negative helical waves partake in the delineation of the AI domain.

Finally, the effect of axial shear and advection on the instability characteristics of purely swirling vortex sheets is more clearly brought out by considering the alternate set of control parameters defined in Eq. (3). Two typical state diagrams in the $\bar{u}-\delta u$ plane are illustrated in Fig. 5 for different centrifugally destabilizing settings of $\delta \gamma$. Consider first $\delta \gamma=1.5$ [Fig. 5(a)]. When $\delta u=0$, there is a narrow AI interval of $\bar{u}$ necessarily associated with finite counterflow. As axial shear $\delta u$ is increased, this AI-interval continuously widens: Larger magnitudes of the advection parameter $\bar{u}$ are then required to return to CI. When $\delta \gamma=0.01$ [Fig. 5(b)], the

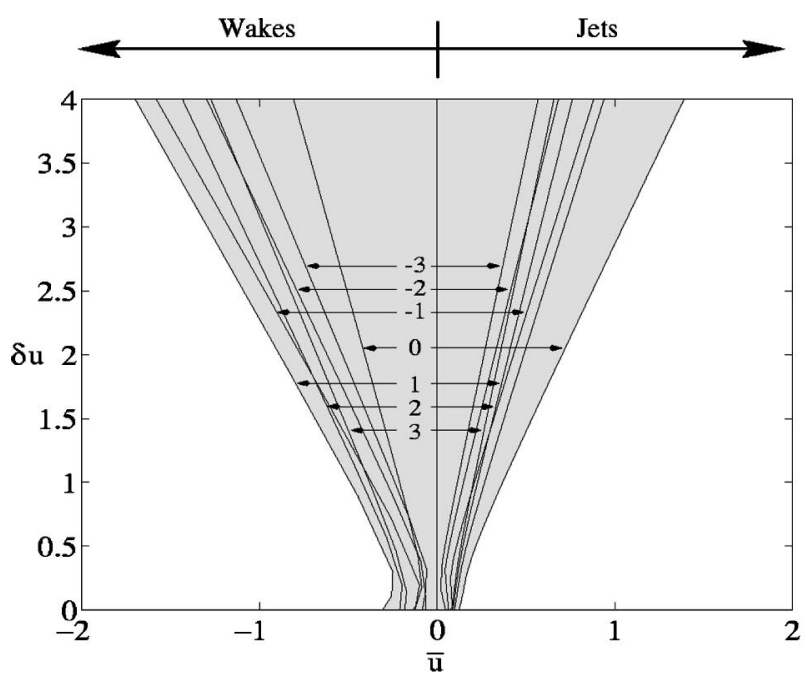

(a)

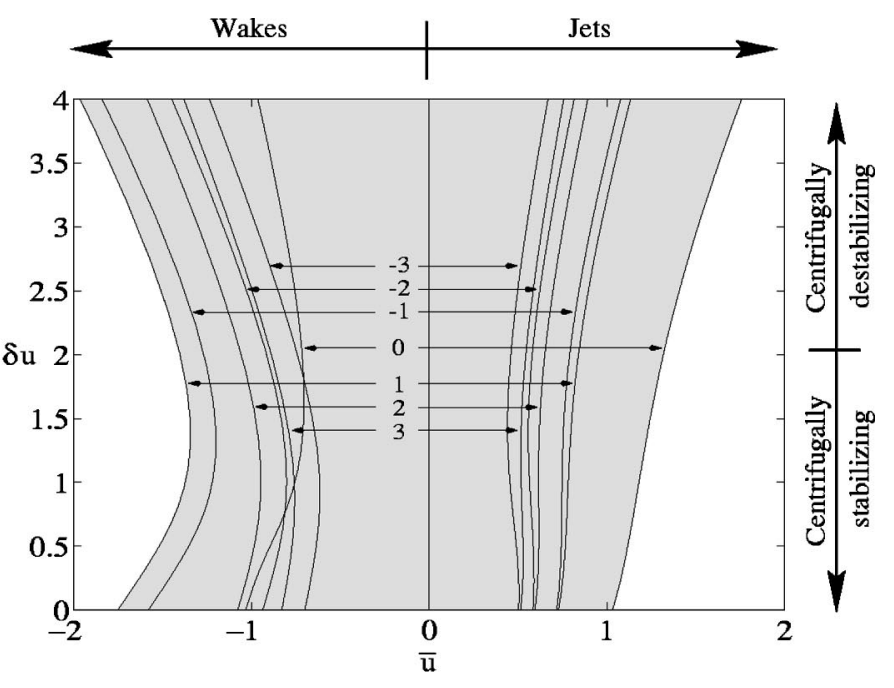

(b)

FIG. 5. AI/CI domains in the $\bar{u}-\delta u$ plane for (a) $\delta \gamma=1.5$ and (b) $\delta \gamma=0.01$. The shaded area corresponds to the absolute unstable region, and the clear area to the convectively unstable region. 
basic flow is only marginally centrifugally destabilizing but the AI-domain extends over a wider range of $\bar{u}$. The same qualitative tendencies are observed in both cases: The critical transitional modes are $m=0$ and $m=1$ on the jet and wake sides, respectively.

\section{CONCLUDING REMARKS}

These results complement those of Lim and Redekopp ${ }^{15}$ in several important respects: A systematic study of the $\mathrm{AI} / \mathrm{CI}$ transition curves pertaining to all helical modes has been conducted for both jets and wakes and for both centrifugally destabilizing and stabilizing swirl distributions.

In the case of jets $(a>-0.5)$, both investigations find that the axisymmetric mode $m=0$ first becomes AI for centrifugally destabilizing swirl configurations. The present analysis further establishes that $m=0$ is replaced by $m$ $=+\infty$ at low mean swirl $\left(\gamma_{c}+\gamma_{\infty}\right) / 2$. Furthermore, this study demonstrates that centrifugally stabilizing swirl triggers a transition to AI via $m=-\infty$.

In the case of wakes $(a<-0.5)$, Lim and Redekopp ${ }^{15}$ find that a centrifugally destabilizing swirl induces AI via the $m=-2$ retrograde mode, whereas we determine that AI first takes place through the $m=+1$ cograde mode, as discussed in Sec. III. Furthermore, the present study shows that centrifugally stabilizing swirl leads to AI via various negative helical modes.

In Ref. 15, the onset of AI in swirling flows is primarily related to the vortex core size: "The more concentrated the vortical core of the swirling flow compared to the jet diameter, the greater the tendency toward absolute instability" ( $\mathrm{p}$. 184 of Ref. 15). In the present context, we prefer to relate the onset of AI to the centrifugally stabilizing or destabilizing nature of the swirl distribution. More specifically, it has been found illuminating to choose as pertinent control parameters the swirl difference $\gamma_{c}-\gamma_{\infty}$, the sign of which determines centrifugal instability or stability, and the mean swirl $\left(\gamma_{c}\right.$ $\left.+\gamma_{\infty}\right) / 2$. As demonstrated in Fig. 4(b) AI is promoted by increasing $\left|\gamma_{c}-\gamma_{\infty}\right|$ on both the centrifugally stabilizing and destabilizing sides. But the centrifugally stabilizing or destabilizing nature of the swirl, i.e., the sign of $\gamma_{c}-\gamma_{\infty}$, leads to radically distinct transitional helical modes.

According to Fig. 4(b), rotation whether centrifugally stabilizing or destabilizing leads to a widening of the AI region, to the extent that co-flow jets $(a>0)$ and wakes $(a<-1)$ may become AI for sufficiently large $\left|\gamma_{c}-\gamma_{\infty}\right|$. Thus, the same general tendency is observed as in the case of a solid body rotation, for instance the centrifugally stabilizing Rankine vortex (Fig. 20 of Ref. 6) or the centrifugally stabilizing Batchelor vortex (Fig. 12 of Ref. 4 and Fig. 7 of Ref. 5). Note, however, that the critical transitional modes are radically different. In the centrifugally stabilizing region $\gamma_{c}-\gamma_{\infty}<0$ of Fig. 4(b), AI/CI transition is dominated by negative helical modes $(m=-1, m=-2, m=-3, \ldots)$ on both the jet and wake sides, as in the Rankine and Batchelor vortices. In the centrifugally destabilizing case $\left[\gamma_{c}-\gamma_{\infty}>0\right.$ of Fig. 4(b)], AI/CI transition solely involves the axisymmetric mode $m=0$ on the jet side and the bending mode $m=1$ on the wake side.

The present study further confirms that, in contrast with purely temporal analyses, $\mathrm{AI} / \mathrm{CI}$ transition criteria lead to the selection of specific helical modes in simple swirling flow profiles. The peculiar $m=-\infty$ transitional mode on the centrifugally stabilizing jet side $\left[\gamma_{c}-\gamma_{\infty}<0, a>-0.5\right.$ in Fig. 4(b)] is likely to be quenched for more realistic basic flow distributions exhibiting a finite shear layer thickness.

The broadening of the AI region and the triggering of an absolute instability above a critical swirl level is a common robust feature of all swirling flow models that have so far been investigated, whether centrifugally stabilizing or not. In the same way, vortex breakdown is also observed in a wide variety of configurations (leading-edge and wing-tip vortices, swirling jets, etc.) but a direct causal link with absolute instability remains to be established on experimentally measured velocity profiles.

${ }^{1}$ R. J. Briggs, Electron-stream Interaction with Plasmas (MIT, Cambridge, Massachusetts, 1964).

${ }^{2}$ A. Bers, "Linear waves and instabilities," in Physique des Plasmas, edited by C. DeWitt and J. Peyraud (Gordon and Breach, New York, 1975), pp. 117-213.

${ }^{3}$ C. Olendraru, A. Sellier, M. Rossi, and P. Huerre, "Absolute/convective instability of the Batchelor vortex," C. R. Acad. Sci. Paris 323-IIb, 153 (1996).

${ }^{4}$ I. Delbende, J.-M. Chomaz, and P. Huerre, "Absolute/convective instabilities in the Batchelor vortex: a numerical study of the linear impulse response,'” J. Fluid Mech. 355, 229 (1998).

${ }^{5}$ C. Olendraru, A. Sellier, M. Rossi, and P. Huerre, "Inviscid instability of the Batchelor vortex: absolute-convective transition and spatial branches," Phys. Fluids 11, 1805 (1999).

${ }^{6}$ T. Loiseleux, J.-M. Chomaz, and P. Huerre, "The effect of swirl on jets and wakes: Linear instability of the Rankine vortex with axial flow," Phys. Fluids 10, 1120 (1998).

${ }^{7}$ According to Rayleigh's circulation criterion (Ref. 8), a necessary and sufficient condition for stability to axisymmetric disturbances of a purely swirling flow is that the square of the circulation does not decrease as the radius is increased.

${ }^{8}$ P. G. Drazin and W. H. Reid, Hydrodynamic Stability (Cambridge University Press, Cambridge, 1981).

${ }^{9}$ P. I. Singh and M. Uberoi, "Experiments on vortex stability,', Phys. Fluids 19, 12 (1976).

${ }^{10}$ P. Billant, J.-M. Chomaz, and P. Huerre, “'Experimental study of vortex breakdown in swirling jets," J. Fluid Mech. 376, 183 (1998).

${ }^{11}$ M. G. Hall, Proceedings of the Heat Transfer and Fluid Mechanics Institute (Stanford U. P., Stanford, 1967), p. 319.

${ }^{12}$ J. E. Martin and E. Meiburg, "On the stability of the swirling jet shear layer," Phys. Fluids 6, 424 (1994).

${ }^{13}$ D. J. Kang and S. P. Lin, "Breakup of a swirling liquid jet," Int. J. Eng. Fluid Mech. 2, 47 (1989).

${ }^{14}$ Z. W. Lian and S. P. Lin, "Breakup of a liquid jet in a swirling gas," Phys. Fluids A 2, 2134 (1990).

${ }^{15}$ D. W. Lim and L. G. Redekopp, "Absolute instability conditions for variable density, swirling jet flows," Eur. J. Mech. B/Fluids 17, 165 (1998).

${ }^{16} \mathrm{P}$. Huerre and P. A. Monkewitz, "Local and global instabilities in spatially developing flows," Annu. Rev. Fluid Mech. 22, 473 (1990). 